\title{
Interaction Potential in Nematogenic 6CHBT ${ }^{\dagger}$
}

\author{
R. B. Bogoslovov, C. M. Roland, ${ }^{*}$, J. Czub, ${ }^{\S}$ and S. Urban ${ }^{\S, \|}$ \\ Chemistry Division, Code 6120, Naval Research Laboratory, Washington, D.C. 20375-5342, and Institute of \\ Physics, Jagiellonian University, Reymonta 4, 30-059 Krakow, Poland
}

Received: June 19, 2008; Revised Manuscript Received: August 19, 2008

\begin{abstract}
Pressure-volume-temperature (PVT) measurements were carried out on the nematic liquid crystal 4(trans$4^{\prime}$-n-hexylcyclohexyl)isothiocyanatobenzene (6CHBT). In combination with previous dielectric relaxation measurements at elevated pressure and new measurements extending to $\mathrm{GHz}$ frequencies, the characteristics of the anisotropic interaction potential were determined. The thermodynamic potential parameter, $\Gamma$, which measures the variation of the interaction energy with volume, equals $5.03 \pm 0.06$, with a barrier height equal to $\sim 7 \mathrm{~kJ} / \mathrm{mol}$. Thus, there is a low potential barrier to reorientations of the $6 \mathrm{CHBT}$ molecule about its short axis; however, the retarding potential is strongly volume-dependent. The longitudinal reorientational times determined for various thermodynamic conditions superpose using a scaling exponent equal to $\Gamma$ within the experimental error. It then follows, as found recently for other liquid crystals, that this relaxation time must be constant along the pressure-dependent clearing line. Thus, the control parameter (e.g., Gibbs free energy) governing the competition between the anisotropic energy and the entropy must also govern the rotational dynamics in the ordered phase of this liquid crystal.
\end{abstract}

\section{Introduction}

The longitudinal relaxation time, $\tau_{\|}$, characterizes molecular reorientation about the short axis (end-over-end rotations) in liquid crystalline (LC) phases consisting of rod-like molecules. ${ }^{1-3}$ These motions are very sensitive to hydrostatic pressure because of coupling to translational movements of neighboring molecules. In the crystal-like phases (smectic $\mathrm{B}, \mathrm{E}, \ldots)$, the distances between mass centers of the parallel layered molecules are considerably shorter than the molecular lengths; thus, rotational jumps can be realized only when fluctuations of the surrounding molecules create sufficient free space. ${ }^{4,5}$ A similar situation is expected to prevail in the nematic $(\mathrm{N})$ phase. Measurement of $\tau_{\|}$as functions of temperature $T$, pressure $P$, and specific volume $V$ (inverse of mass density) is essential to understanding the nature of the molecular interactions in LC phases..$^{3,6-11}$ In particular, the effective steepness of the interaction potential in the LC phase can be determined from analysis of $\tau_{\|}$at different thermodynamic conditions. Since mesogenic materials have appreciable dipole moments (anisotropic interactions being the origin of the liquid crystallinity), dielectric spectroscopy is an especially effective technique for such experiments.

According to the mean-field theory of liquid crystals, ${ }^{12}$ the product $T_{c} V_{c}^{\Gamma}$ is a constant, where $T_{c}$ and $V_{c}$ refer to the respective temperature and specific volume along the pressuredependent phase boundary and the thermodynamic potential parameter is defined as

$$
\Gamma=-\left(\frac{d \log T_{c}}{d \log V_{c}}\right)_{P}
$$

This parameter appears in the orientation dependent part of the potential, $u \propto V^{-\Gamma}$, and is a measure of the strength of the

\footnotetext{
$\dagger$ Part of the "Karl Freed Festschrift".

* Corresponding author. E-mail: roland@nrl.navy.mil.

¥ Naval Research Laboratory.

$\S$ Jagiellonian University.

"E-mail: ufurban@cyf-kr.edu.pl.
}

steric repulsions relative to the attractive part of the potential. $\Gamma$ was introduced by Maier and Saupe in their model of the nematic state ${ }^{12}$ and subsequently applied to other LC phase transitions. ${ }^{11,13-16} P V T$ data for liquid crystalline materials through their transition make possible determination of $\Gamma$.

Equation 1 relates to the equilibrium phase boundaries of the ordered state. A method of analyzing the dynamics is based on the relation

$$
\tau(T, V)=f\left(T V^{\prime}\right)
$$

proposed by Casalini and Roland ${ }^{17,18}$ for the reorientational relaxation time, $\tau$, of isotropic glass-forming liquids (or the corresponding local segmental relaxation time of polymers); here, $\gamma$ is a material constant and $f$ is a function. It has been demonstrated for about 50 liquids that eq 2 allows rescaling of relaxation data obtained at different thermodynamic conditions to a common master curve using a single adjustable parameter $\gamma \cdot{ }^{18,19}$ Moreover, molecular dynamics simulations of LennardJones particles have shown that the value of $\gamma$ reflects the steepness of the repulsive core of the intermolecular potential in the range dominating the structural dynamics. ${ }^{20}$

Equation 2 was recently applied to the longitudinal relaxation times of several LC substances measured under different thermodynamic conditions. ${ }^{9-11}$ For LC the superposition of $\tau_{\|}$ is very accurate, with $f$ assuming a simple exponential form

$$
\tau_{\|}(T, V)=\tau_{0} \exp \left(C / T V^{\gamma}\right)
$$

where $\tau_{0}$ and $C$ are constants. This differs from the scaling behavior of isotropic liquids, for which plots of $\log \tau$ versus $T^{-1} V^{-\gamma}$ are nonlinear. ${ }^{18}$ The Arrhenius nature of the scaled data for LC implies that $V^{\gamma}$ reflects the volume-dependence of the height of the activation barrier.

The two material constants, $\Gamma$ and $\gamma$, would be equal if $\tau_{\|}$ were constant along the transition line $T_{c}(P) .{ }^{11}$ These two approaches to describing the steepness of the intermolecular potential are employed in the present paper, in which we report $P V T$ data for the nematogenic substance $6 \mathrm{CHBT}$, 


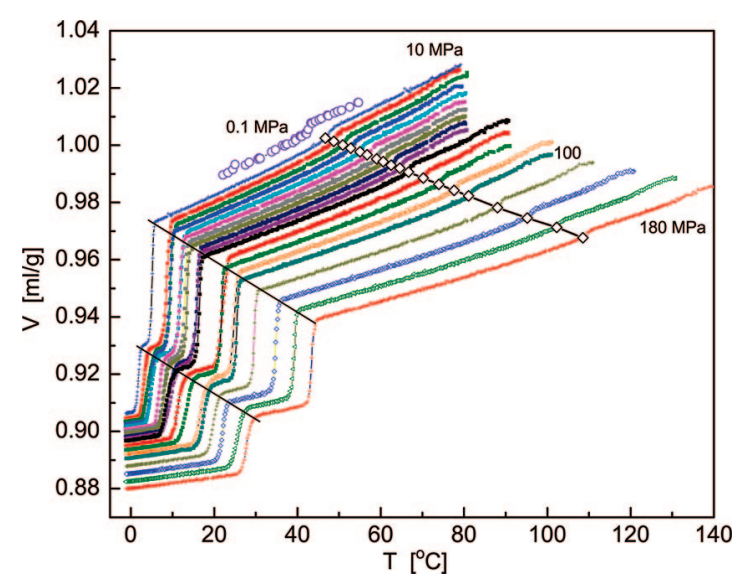

Figure 1. Specific volume versus temperature for 6CHBT (chemical structure shown) at pressures $=0.1 \mathrm{MPa}$, from 10 to $50 \mathrm{MPa}$ in steps of $5 \mathrm{MPa}$, from 50 to $100 \mathrm{MPa}$ in steps of $10 \mathrm{MPa}$, and from 100 to $180 \mathrm{MPa}$ in steps of $20 \mathrm{MPa}$. The temperatures and specific volumes at the clearing transition, denoted by the open diamonds, are replotted in Figure 3.

4(trans-4'-n-hexylcyclohexyl)isothiocyanatobenzene (structure in Figure 1). This compound has been studied previously under elevated pressure by Hartmann et al. ${ }^{21}$ who determined the phase diagram, and by Urban and Würflinger, ${ }^{22}$ who reported the dielectric properties. The present determination of the density as a function of pressure and temperature $(P V T)$, in combination with new dielectric measurements in the $10^{6}-10^{9} \mathrm{~Hz}$ regime (i.e., above the clearing point), completes the characterization of 6CHBT and enables an analysis that takes into account existing theoretical approaches to liquid crystallinity.

\section{Experimental Section}

The 6CHBT sample was synthesized by R. Dabrowski at the Military University of Technology, Warsaw, Poland. ${ }^{23,24}$ The nematic phase at atmospheric pressure exists between $13.3{ }^{\circ} \mathrm{C}$ (melting point) and $43.5^{\circ} \mathrm{C}$ (clearing point). The volume change of a $\sim 1 \mathrm{~mL}$ sample was measured as a function of pressure and temperature with a Gnomix instrument, utilizing mercury as the confining fluid. At each pressure the sample was cooled at $0.5 \mathrm{C} / \mathrm{min}$ from above the clearing temperature to $-16^{\circ} \mathrm{C}$. For the ensuing isobaric run, the pressure was raised prior to increasing the temperature to the initial value; the latter was in the range from 90 to $140{ }^{\circ} \mathrm{C}$ depending on the pressure. The differential volume data were converted to specific volume using the absolute value determined at ambient conditions. High frequency dielectric spectra $(1 \mathrm{MHz}$ to $1.8 \mathrm{GHz}$ ) were obtained at ambient pressure with an HP 4291A impedance analyzer. The sample was contained between gold-plated electrodes in a parallel plate configuration $(7 \mathrm{~mm}$ diameter $\times 0.85 \mathrm{~mm}$ thickness). Temperature control employed an ESPEC SH-240 chamber with $\sim 0.1{ }^{\circ} \mathrm{C}$ stability. Spectra were measured over a temperature range from 24.6 to $84.1{ }^{\circ} \mathrm{C}$, which encompasses the nematic to isotropic transition. A 60 min equilibration time was used at each temperature.

\section{Results and Discussion}

The variation in the specific volume with temperature for various pressures is shown in Figure 1, with three phase transitions evident. These transitions are also observed in DTA measurements ${ }^{21}$ and Figure 2 compares the two. Only the transition temperatures for the isotropic-nematic phase

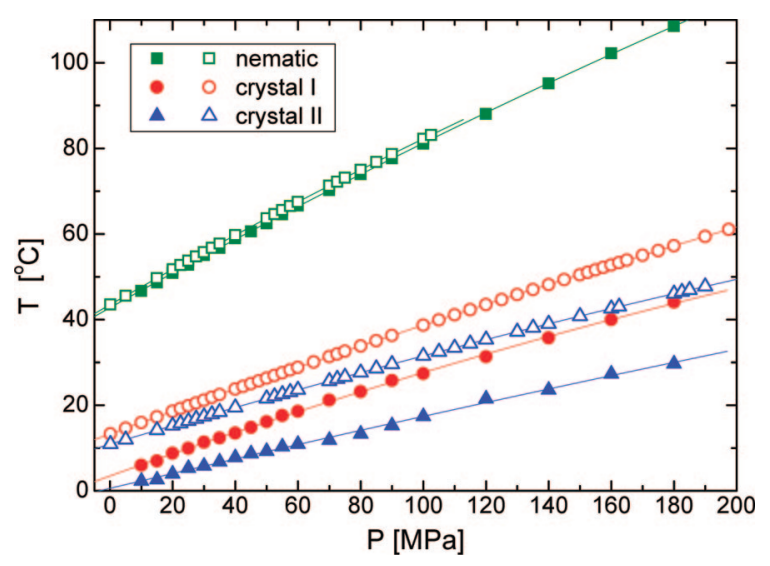

Figure 2. Pressure-temperature phase diagram for $6 \mathrm{CHBT}$ determined from DTA ${ }^{21}$ (open symbols) and from PVT (filled symbols) measurements for the nematic and the two crystalline phases.

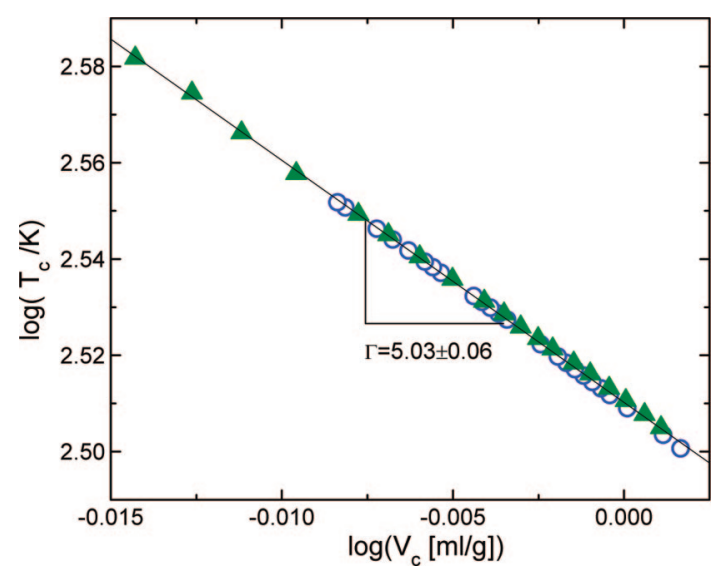

Figure 3. Double logarithmic plot of the clearing temperature versus volume for 6CHBT from measurements by DTA (open symbols) and PVT (filled symbols) at various pressures. The negative slope yields the indicated value for the thermodynamic potential parameter.

transition agree well. This is a consequence of differences in thermal histories (DTA obtained during heating and PVT during cooling) and consequent differences in supercooling. Although the transitions involving a crystalline phase (nematic-crystal I and crystal I-crystal II) are influenced considerably by supercooling, the transition lines are parallel, indicating that the pressure dependences are not affected by thermal history.

Figure 3 shows double logarithmic plots of $T_{c}$ versus $V_{c}$ (i.e., along the clearing line separating the nematic and isotropic phases of 6CHBT). Included are both the PVT and the DTA data, specific volumes for the latter calculated from the equation of state fit to the PVT measurements for the nematic phase:

$$
\begin{array}{r}
V=0.9741 \exp \left(6.90 \times 10^{-4} T\right)[1-0.894 \ln (1+ \\
\left.\left.\frac{P}{205.8 \exp (-0.00467 T)}\right)\right](4)
\end{array}
$$

with $V$ in $\mathrm{mL} / \mathrm{g}, T$ in Celsius, and $P$ in $\mathrm{MPa}$. Fitting the combined data sets in Figure 3 to eq 1 gives $\Gamma=5.03 \pm 0.06$. This is toward the high side of the range found for various LC materials..$^{3,15,25}$ However, from the behavior of other liquid crystals, ${ }^{11}$ we anticipate $\Gamma$ to be consistent with the value of the scaling exponent for $\tau_{\|}$.

In ref 22 , the dielectric $\tau_{\|}$were analyzed for two thermodynamic conditions: isobaric, yielding the activation enthalpy $\Delta^{\#} H$ 

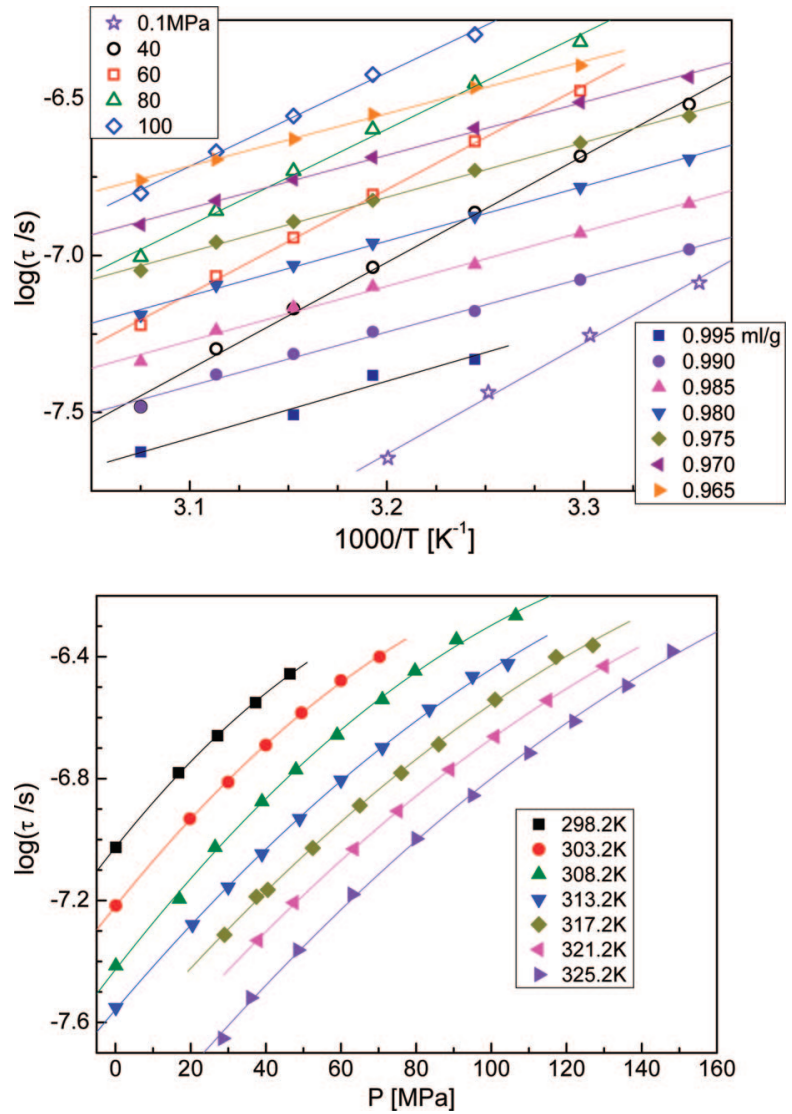

Figure 4. Activation plots for the nematic phase of 6CHBT for (top) isobaric and isochoric conditions and (bottom) isothermal conditions.

$=R\left(\partial \ln \tau_{\|} / \partial T^{-1}\right)_{P}=63 \pm 2 \mathrm{~kJ} / \mathrm{mol}$, and isothermal, yielding the activation volume $\Delta^{\#} V=R\left(\partial \ln \tau_{\|} / \partial \mathrm{P}\right)_{T}=20 \mathrm{~mL} / \mathrm{mol}(R$ is the gas constant). From the $P V T$ data, we calculate the activation energy for several isochors, obtaining $\Delta^{\#} U=R\left(\partial \ln \tau_{\mid /} / \partial T^{-1}\right)_{V}=$ $33 \pm 2 \mathrm{~kJ} / \mathrm{mol}$. These activation plots are shown in Figure 4. The ratio $\Delta^{\#} U / \Delta^{\#} H$ is a measure of the relative magnitude of thermal and volume effects on $\tau_{\|}$; we obtain $\Delta^{\#} U / \Delta^{\#} H=0.52$ \pm 0.03 . Since the activation enthalpy, comprising both thermal and volume contributions, is roughly twice the activation energy, which includes only the thermal effects, this indicates a near equivalent influence of the two thermodynamic quantities on the hindrance of longitudinal molecular motions. This is in accord with the general behavior of LC phases in the nematic phase. ${ }^{3,7-10}$ Similar results have been obtained for the reorientations in supercooled liquids and polymers, although the ratio tends to be somewhat larger for the latter (i.e., volume effects are weaker in polymers). ${ }^{18,26}$

If eq 2 applies, there is a simple relation connecting the activation energy ratio and the scaling exponent $\gamma^{17}$

$$
\frac{\Delta^{\#} U}{\Delta^{\#} H}=\left(1+\alpha_{P} T \gamma\right)^{-1}
$$

Using $\alpha_{P}=5.76 \times 10^{-4} \mathrm{~K}^{-1}$ at $T_{\mathrm{c}}=316.7 \mathrm{~K}$, eq 5 gives $\gamma=$ $5.1 \pm 0.3$, in accord with the obtained $\Gamma(=5.0)$. This is the value of $\gamma$ that should superpose the longitudinal relaxation times (eq 2). As shown in Figure 5, the data in Figure 4 for different thermodynamic conditions rescale to a single master curve, with the smallest scatter of points achieved for $\gamma=5$.0. This $\gamma$ provides a measure of the steepness of the repulsive core, which reflects both the repulsive and the attractive contributions to the intermolecular potential. ${ }^{19,20,27}$

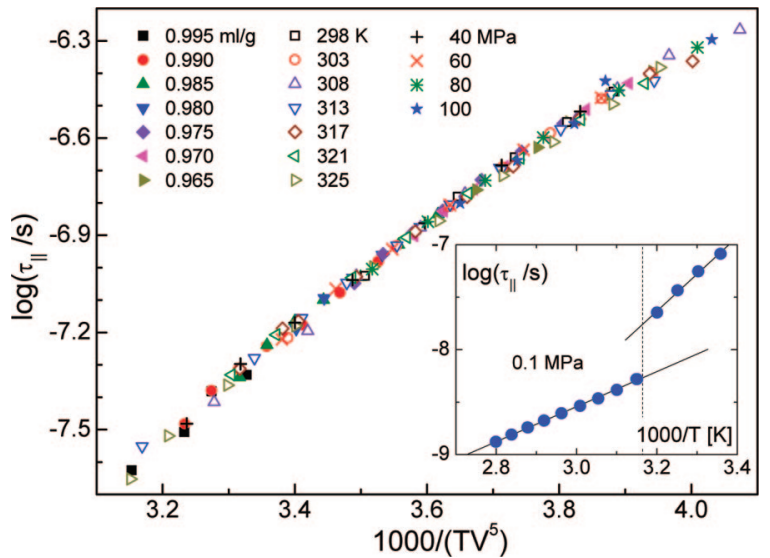

Figure 5. Thermodynamical scaling of the longitudinal relaxation time in the nematic phase of 6CHBT. The inset shows the measurements at ambient pressure used to determine the retardation factor, $g=3.24 \pm$ 0.15 .

Another characteristic of the intermolecular potential is the height of the energy barrier, $\sigma$

$$
U(\theta)=-R T \sigma P_{2}(\theta)
$$

where $P_{2}(\theta)$ is the second Legendre polynomial and $\theta$ is the orientation angle. The energy barrier is related to $\Gamma$ by $^{3}$

$$
\sigma=-v_{0} V^{-\Gamma} S
$$

In this relation, $v_{0}$ is a constant and $S$ is the order parameter (average value of $P_{2}(\theta)$ ). For nematics $\sigma$ (in units of RT) is related to the retardation factor, $g$, according to ${ }^{1,28}$

$$
g=\frac{e^{\sigma}-1}{\sigma}\left(\frac{2 \sigma^{3 / 2}}{\pi^{1 / 2}(1+\sigma)}+2^{-\sigma}\right)^{-1}
$$

The retardation factor is an experimentally measurable quantity, defined as ${ }^{1}$

$$
g=\tau_{\mathrm{II}} / \tau_{\text {iso }}
$$

where $\tau_{\text {iso }}$ is the relaxation time for the isotropic liquid; thus, $g$ characterizes the reduction in mobility due to the mesogenic ordering. For the 6CHBT, we obtain $g=3.24 \pm 0.15$ for the clearing point at ambient pressure (see inset to Figure 5), somewhat smaller than a previously reported value of $4.4 .^{29}$ Equation 8 then gives $\sigma=2.6(=7 \mathrm{~kJ} / \mathrm{mol}$ at the clearing point), implying a weak potential barrier. This result is consistent with the magnitude of the scaling exponent, $\gamma=5$, which is larger than those obtained for similar nematogenic $n \mathrm{PCH}$ compounds (having the $\mathrm{CN}$ group instead of NCS). ${ }^{9}$ On the other hand, potential barriers for $n \mathrm{PCHs}^{30}$ are close to the value for $6 \mathrm{CHBT}$. The $\gamma$ for 6CHBT is also large in comparison to alkylisothiocyanato-biphenyl liquid crystals, for which $\gamma$ is in the range $2-3 .^{11}$

The equivalence of the thermodynamical (i.e., $\Gamma$ ) and dynamical (i.e., $\gamma$ ) parameters for the nematogenic 6CHBT affirms the connection between the rotational motions and the repulsive part of the interaction potential. It also means that the longitudinal relaxation time must be invariant to thermodynamic conditions $(T, P$, and $V$ ) along the clearing line; that is, $\tau_{\|}(P)_{T_{c}}$ is a constant. Since the relaxation times 
were not measured at temperatures that high for elevated pressures, we extrapolate the data shown in Figure 5 using

$$
\log \tau_{\|}=-1.46-0.019 \times T V^{5}
$$

The mean value at the clearing point for all measured pressures was $T_{c} V_{c}^{5}=324.1 \pm 0.3$, which gives $\log \left(\tau_{\|} / \mathrm{s}\right)=$ $-7.76 \pm 0.01$. This is in good agreement with the value measured at ambient pressure (Figure 5 inset), indicating that the magnitude of the relaxation time at the nematic-isotropic transition is indeed constant.

This result is not predicted by theories for LC materials. However, both mean field theory ${ }^{12}$ and experiments indicate that the order parameter attains a value at the nematic-isotropic transition that is constant ${ }^{13,31}$ or decreases slightly with pressure. ${ }^{31,32}$ Since $S$ can be related to the retardation factor through the strength of the nematic potential, ${ }^{1,2}$ it follows from the constancy of $\tau_{\|}(P)$ that $\tau_{\text {iso }}$ should also be essentially a constant along the clearing line.

\section{Conclusions}

Equation of state data through the clearing line were used to determine the thermodynamic potential parameter. The result, $\Gamma=5.03 \pm 0.06$, is consistent with the value of the scaling exponent, $\gamma=5.1 \pm 0.3$, that superposes the longitudinal relaxation times. From the retardation factor measured at ambient pressure, eq 7 yields a value for the energy barrier $=$ $7 \mathrm{~kJ} / \mathrm{mol}$. This small magnitude is consistent with a strong volume dependence, reflected in the large value of $\Gamma$ (viz. eq 7 ). Thus, in 6CHBT the potential barrier is low but is markedly affected by volume changes.

The equivalence of $\Gamma$ and $\gamma$ means that the longitudinal relaxation time must be a constant at the pressure-dependent clearing temperature; we find $\tau_{\|}(P)_{T_{c}}=17.4 \pm 0.4 \mathrm{~ns}$. That the thermodynamic conditions associated with the stability limits of the ordered state (reflecting competition between the anisotropic interaction energy and the orientational entropy) bear a direct relationship to the time scale of molecular rotations is unanticipated by any model and should guide theoretical progress in this important class of materials.

Acknowledgment. The work at the Naval Research Laboratory was supported by the Office of Naval Research. R.B. acknowledges the American Society for Engineering Education for a postdoctoral fellowship.

\section{References and Notes} 119.

(1) Martin, A. J.; Meier, G.; Saupe, A. Symp. Faraday. Soc. 1971, 5,

(2) Kalmykov, Y. P.; Coffey, W. T. Liq. Cryst. 1998, 25, 329.

(3) Urban, S.; Würflinger, A. Adv. Chem. Phys. 1997, 98, 143.

(4) Urban, S.; Czub, J.; Przedmojski, J.; Dbrowski, R.; Geppi, M. Mol. Cryst. Liq. Cryst. 2007, 477, 87.

(5) Jasiurkowska, M.; Budziak, A.; Czub, J.; MassalskaArod, M.; Urban, S. Liq. Cryst. 2008, 35, 513.

(6) Bates, M. A.; Luckhurst, G. R. Mol. Phys. 2001, 99, 1365.

(7) Würflinger, A.; Urban, S. In Relaxation Phenomena; Haase, W., Wróbel, S., Eds.; Springer, New York, 2003; Chapter 1.4.

(8) Reference 7, Chapter 4.2.

(9) Urban, S.; Würflinger, A. Phys. Rev. E 2005, 72, 021707.

(10) Urban, S.; Roland, C. M.; Czub, J.; Skrzypek, K. J. Chem. Phys. 2007, 127, 094901.

(11) Roland, C. M.; Bogoslovov, R. B.; Casalini, R.; Ellis, A. R.; Bair, S.; Rzoska, S. J.; Czupryñski, K.; Urban, S. J. Chem. Phys. 2008, 128, 224506.

(12) Maier, W.; Saupe, A. Z. Naturforsch. 1958, 13a, 564. 1959,14a, 882; 1960, 15a, 287.

(13) McColl, J. R.; Shih, C. S. Phys. Rev. Lett. 1972, 29, 85.

(14) Johnson, C. S.; Collings, P. J. J. Chem. Phys. 1983, 79, 4056.

(15) Würflinger, A.; Sandmann, M. In Physical Properties of Liquid Crystals: Nematics; Dunmur, D.A., Fukuda, A., Luckhurst, G. R., Eds.;EMIS DataReview Series, 25; Inst. Elec. Eng., London, 2001; p 151A.

(16) Würflinger, A.; Urban, S. Phys. Chem. Chem. Phys. 2001, 3, 3727.

(17) Casalini, R.; Roland, C. M. Phys. Rev. E 2004, 69, 062501.

(18) Roland, C. M.; Hensel-Bielowka, S.; Paluch, M.; Casalini, R. Rep. Prog. Phys. 2005, 68, 1405.

(19) Roland, C. M.; Bair, S.; Casalini, R. J. Chem. Phys. 2006, 125, 124508.

(20) Coslovich, D; Roland, C. M. J. Phys. Chem. B 2008, 112, 1329.

(21) Hartmann, M.; Jenau, M.; Würflinger, A.; Godlewska, M.; Urban, S. Z. Physik. Chem. 1992, 177, 195.

(22) Urban, S.; Würflinger, A. Liq. Cryst. 1992, 12, 931.

(23) Dbrowski, R.; Dziaduszek, J.; Szczuciñski, T. Mol. Cyst. Liq. Cryst. Lett. 1984, 102, 155; Mol. Cyst. Liq. Cryst. 1985, 124, 241.

(24) Baran, J. W.; Raszewski, Z.; Dbrowski, R.; Kêdzierski, J.; Rutkowska, J. Mol. Cyst. Liq. Cryst. 1985, 123, 237.

(25) Chandrasekhar, S. Liquid Crystals 2nd Ed.; Cambridge Univ. Press: United Kingdom, 1992.

(26) Roland, C. M.; Feldman, J. L.; Casalini, R. J. Non-Cryst. Solids 2006, 352,4895 .

(27) Pedersen, U. R.; Bailey, N. P.; Schrøder, T. B.; Dyre, J. C. Phys. Rev. Lett. 2008, 100, 015701.

(28) Coffey, W. T.; Crothers, D. S. F.; Kalmykov, Y. P.; Waldron, J. T. Physica A 1995, 213, 551.

(29) Urban, S.; Czuprynski, K.; Dabrowski, R.; Gestblom, B.; Janki, J.; Kresses, H.; Schmalfuss, H. Liq. Cryst. 2001, 28, 691.

(30) Urban, S.; Gestblom, B.; Würflinger, A. Mol. Cryst. Liq. Cryst. 1999, 331, 113.

(31) Wallis, G. P.; Roy, S. K. J. Phys. (Paris) 1980, 41, 1165.

(32) Horn, R. G.; Faber, T. E. Proc. R. Soc. London A 1979, 368, 199.

JP805421G 